to identify over-users and measure response to treatment.

Pippa Murdie

Original article Ferrari A et al. (2005) Need for analgesics/ drugs of abuse: a comparison between headache patients and addicts by the Leeds Dependence Questionnaire (LDQ). Cephalalgia [doi: 10.1111/j.1468-2982.2005.01020.x]

\section{Varicella vaccination program: a success?}

Following chickenpox, the varicella zoster virus remains latent in the nervous system; its reactivation causes the neurological disorder herpes zoster (shingles). Before varicella vaccine licensing in the US in 1995, most people developed chickenpox, with $\sim 13,000$ hospitalizations and 100-150 deaths annually. A group from the Centers for Disease Control and Prevention studied the impact of this vaccine on hospitalization and related costs.

This retrospective, population-based study used data from MarketScan ${ }^{\circledR}$ databases (MEDSTAT Group, Inc., Ann Arbor, MI) covering 40 employer insurance plans-about 4 million covered lives per year. The study sample included patients aged 0-49 years who had hospitalizations/ambulatory visits caused by varicella between 1994 (prevaccination) and 2002. Patients were divided into three groups: $<10$ years, $10-19$ years and $20-49$ years. Infants ( $<1$ year), who are ineligible for vaccination, were also evaluated.

Varicella hospitalizations decreased significantly in all groups between 1994 and 2002 $(P<0.001)$, with the greatest decline among infants. Ambulatory visits with varicella as the primary diagnosis also decreased-from $215 / 100,000$ population in the prevaccination period to 89/100,000 in $2002(P<0.001)$. Declining rates among infants and adults indicate the presence of herd immunity in the population. Hospitalization:ambulatory visit ratios decreased during the study, suggesting decreasing disease severity. Estimated annual medical expenditure from varicella decreased from US\$84.9 million prevaccination, to $\$ 22.1$ million in 2002.

Zhou et al. conclude that the varicella vaccination program has shown considerable success, with a dramatic decrease in varicella hospitalizations, ambulatory visits and related expenditure. As the study population was not fully representative, however, further surveillance is needed to more accurately examine the impact of varicella vaccination.

Rebecca Ireland

Original article Zhou F et al. (2005) Impact of varicella vaccination on health care utilization. JAMA 294: 797-802

\section{Systemic lupus erythematosus and neuropsychiatric syndromes}

Neuropsychiatric syndromes in patients with systemic lupus erythematosus (SLE) might be attributable to non-SLE factors, according to a recent study by Hanly and colleagues. Nervous system involvement is a common feature of SLE; however, few trials have addressed the extent of non-SLE-specific causes of these neuropsychiatric events.

The Canadian study compared the prevalence, diversity and clinical significance of neuropsychiatric syndromes in 53 patients with SLE and 53 patients with rheumatoid arthritis (RA). The demographic features of the two groups were similar.

The trial showed that the number of patients with a neuropsychiatric event was higher in the RA group than the SLE group. With regard to health-related quality of life, fatigue, depression and anxiety, and cognitive dysfunction, there were no significant differences between the two groups. More SLE patients with cumulative neuropsychiatric syndromes, experienced cognitive distress than did RA patients with cumulative neuropsychiatric syndromes, although this did not translate into a lower health-related quality of life.

The finding that anxiety, headache and mood disorders were comparable in both groups is interesting: it suggests that SLE patients are no more likely to experience these symptoms than patients with other chronic rheumatic diseases. The etiology of neuropsychiatric syndromes in patients with SLE is multifactorial, and correctly identifying the cause is a significant challenge. In conclusion, there is sufficient evidence to suggest that in a substantial proportion of patients, neuropsychiatric syndromes are not attributable to SLE.

Rachel Murphy

Original article Hanly JG et al. (2005) Neuropsychiatric syndromes in patients with systemic lupus erythematosus and rheumatoid arthritis. J Rheumatol 32: 1459-1466 